\title{
Smoking Cessation Interventions in the Cardiology Ward
}

\author{
Mark Philip Cassar \\ John Radcliffe Hospital, Oxford
}

\begin{abstract}
Hospital admission is a unique opportunity for a smoking cessation attempt; smokers may be more motivated to quit as they are distanced from the usual cues they face associated with nicotine consumption, and the effect of poor lifestyle habits on their health are brought to light. With most hospitals employing smoke-free grounds, patients are further inclined to stop smoking.
\end{abstract}

According to NICE guidance, smoking cessation support should be delivered within 1 working day of admission for inpatients, and NRT products should be recommended and offered to all people who smoke. Despite this, many smokers on the cardiology ward at the John Radcliffe Hospital fail to receive smoking cessation advice, and are unable to benefit from Nicotine Replacement Therapy. This leads to missed opportunities to stop smoking, increased morbidity in patients undergoing cardiovascular procedures, and increased costs for the NHS, with patients who continue smoking being re-admitted with more smoking-related illnesses in the future.

\section{Problem}

Doctors working in the cardiology ward are excellent at treating patients with the use of cardiac medications and interventions. However, smoking cessation advice is often overlooked. This results in missed opportunities for patients to stop smoking and improve their cardiovascular and overall health.

\section{Background}

A literature review was performed to outline the effects of smoking on health, quantify the burden of disease that nicotine addiction creates, assess the efficacy of nicotine replacement therapy (NRT) and smoking cessation meetings, and determine whether NRT is cost effective. The following is a succinct summary of the information gathered:

1. Smoking causes $84 \%$ of deaths from lung cancer, $83 \%$ of deaths from chronic obstructive disease, 3 out of 10 cancer deaths, and 1 out of every 7 deaths from heart disease.[1]

2. NRT products should be recommended and offered to all smokers admitted to hospital.[2]

3. All frontline staff in hospital should be trained to deliver stop smoking interventions.[2]

4. NRT is more cost-effective than chemotherapy for lung cancer and statins for coronary vascular disease.[3]

After discussion with the trust smoking cessation lead, the barriers to prompt smoking cessation intervention were realised; NRT can only be prescribed after a patient is assessed by a smoking cessation adviser, unless the prescription is endorsed by a consultant.

Previous attempts to allow doctors to prescribe NRT without prior review by a smoking cessation adviser failed. This was due to a potential increase of nicotine replacement prescriptions without appropriate patient referral and follow-up by a smoking cessation adviser.

Therefore, the process of attaining smoking cessation adviser status was clarified; one could attain certification following attendance of a one day training course. For doctors working within the trust, a three hour meeting held in hospital was deemed equivalent, as was completion of an online module on the National Centre for Smoking Cessation and Training (NCSCT) website supplemented by a 30 minute meeting outlining the local policies.

See supplementary file: ds5155.docx - "Smoking QIP"

\section{Baseline measurement}

20 patients, prior to discharge, were asked the following questions:

1. Have you been asked whether you smoke?

2. Do you smoke?

3. Have you been given smoking cessation advice or prescribed NRT?

The results showed that $60 \%$ of patients were asked their smoking status, $40 \%$ of which were active smokers. $50 \%$ of smokers received smoking cessation advice, with only $25 \%$ being prescribed nicotine replacement therapy.

\section{Design}

Once the process of NRT prescription was understood, it became clear that the only way to ensure prompt delivery of a stop smoking attempt was for junior doctors to attain smoking cessation adviser certification. This would equip doctors with the tools required to carry out a smoking cessation assessment and also prescribe NRT. By completing practitioner training on the NCSCT website and attending a 30 minute smoking cessation meeting on site, doctors 
could carry out the majority of the certification process in the comfort of their own home.

Repeat measurements, using 20 patients as a sample size, were carried out to assess results. Further PDSA cycles were designed using the measurements gathered after the previous PDSA cycle was complete.

The project was initiated in September 2014, and measurements were concluded in February 2015.

\section{Strategy}

PDSA Cycle 1 - A presentation on the harmful effects of smoking together with the resources available to help patients stop smoking was delivered.

PDSA Cycle 2 - Senior House Officers completed online practitioner training and attended a smoking cessation adviser meeting. This gave an overview of the NRTs available within the trust and served to inform the doctors on the necessary forms requiring submission to ensure appropriate patient follow-up.

PDSA Cycle 3 - The handover sheet used daily during the morning meeting was updated to incorporate whether each patient had a smoking assessment done. To allow nurses to update the data with ease, senior house officers were encouraged to record smoking status within the electronic patient record.

PDSA Cycle 4 - The Junior Medical Staffing Coordinator was contacted, and together with the smoking cessation lead, NCSCT smoking cessation training was organised and completed by the new group of senior house officers.

\section{Post-measurement}

Following the first PDA cycle, the amount of nicotine replacement prescribed was unchanged. This was due to doctors not recording smoking status, and due to a scarcity of smoking cessation advisers on the ward.

Having attained smoking cessation adviser status, the second PDA cycle allowed doctors to prescribe NRT. Following this cycle, the percentage of patients being asked about their smoking status remained unchanged. However, all the smokers involved in this cycle had NRT prescribed. These resulted may be attributed to poor patient recall as well as poor engagement from doctors.

With the electronic recording of smoking status, misrepresentation of smoking cessation interventions within the ward was amended by eliminating the reliance on patient recall. The flagging of patients at handover who had not yet undergone smoking assessment served as a reminder to doctors to engage with patients and query their status. This led to all patients in the cycle having their smoking status determined, and NRT being prescribed to all smokers.

Training the new group of senior house officers resulted in maintenance of the previously achieved rates of smoking status identification and NRT prescription.

See supplementary file: ds5415.jpg - "Table and Graph depicting measurement results throughout the project"

\section{Lessons and limitations}

The background research performed was essential to identify and avoid the pitfalls previous attempts succumbed to. This led to the implementation of a unique strategy which was ultimately successful.

The main difficulties encountered in the project were twofold; smoking cessation adviser certification needed to be achieved for the project to be successful, and recording of smoking status, together with the prescription of NRT, was mandatory for improvement in patient care. With the bulk of training carried out online, staff were able to achieve certification with no disruption to their daily duties. By demonstrating to staff the ease and minimal time required for smoking status to be recorded on the electronic patient record, the aim of the project was achieved.

In 2015, the licence for the provision of smoking cessation therapies will be transferred to a different provider. This might affect the way smoking cessation products are prescribed (for example, requiring a different route for attainment of smoking cessation adviser status). To sustain the change brought about by this project, a meeting will be held with the relevant stakeholders to clarify the new processes that will be brought in place for prescription of NRT. Due to the impending change, extension of the project to other wards in the hospital will be attempted at a later date (in the 4th quarter of 2015).

\section{Conclusion}

The initial problem at the beginning of the project was clear; NRT was available for inpatients, but was rarely utilised by junior doctors. With the achievement of NCSCT certification by all the senior house officers, the recording of smoking status in the electronic patient record and the flagging up of patients yet to be queried about their smoking status, NRT prescription significantly increased from $25 \%$ to $100 \%$, and this was maintained after rotation of the senior house officers. The inference from this is that in the long term, patients are likely to experience improvements in health, leading to fewer admissions to hospital, both cardiac and non-cardiac related. Despite the theoretical nature of the inference (as to measure hospital re-admissions following this intervention would have been an unrealistic aim to achieve), the knowledge base is clear that NRT yields more successful stop smoking attempts, leading to a cost-effective reduction in the use of NHS resources.

\section{References}

1. Department of Health. A White Paper on Tobacco. London: London: The Stationery Office; 1998.

2. Nice.org.uk. Smoking cessation in secondary care: acute, maternity and mental health services | Guidance and 
guidelines | NICE [Internet]. 2013 [cited 15 September 2015]. Available from:

http://www.nice.org.uk/Guidance/PH48

3. Shahab L. Cost-effectiveness of pharmacotherapy for smoking cessation [Internet]. 1st ed. London; 2012 [cited 14 September 2014]. Available from: http://www.ncsct.co.uk/usr/pub/B7 Costeffectiveness_pharmacotherapy.pdf

\section{Declaration of interests}

\section{None}

\section{Ethical approval}

Approval was granted from the consultants in the cardiology department. 